\title{
On the Two Main Laws of Thermodynamics
}

\author{
Martina Costa Reis and Adalberto Bono Maurizio Sacchi Bassi \\ Universidade Estadual de Campinas \\ Brazil
}

\section{Introduction}

The origins of thermodynamics date back to the first half of the nineteenth century, when the industrial revolution occurred in Europe. Initially developed for engineers only, thermodynamics focused its attention on studying the functioning of thermal machines. Years after the divulgation of results obtained by Carnot on the operation of thermal machines, Clausius, Kelvin, Rankine, and others, re-discussed some of the ideas proposed by Carnot, so creating classical thermodynamics. The conceptual developments introduced by them, in the mid of XIX century, have allowed two new lines of thought: the kinetic theory of gases and equilibrium thermodynamics. Thus, thermodynamics was analyzed on a microscopic scale and with a mathematical precision that, until then, had not been possible (Truesdell, 1980). However, since mathematical rigor had been applied to thermodynamics through the artifice of timelessness, it has become a science restricted to the study of systems whose states are in thermodynamic equilibrium, distancing itself from the other natural sciences.

The temporal approach was resumed in the mid-twentieth century only, by the works of Onsager (Onsager, 1931a, b), Eckart (Eckart, 1940) and Casimir (Casimir, 1945), resulting in the thermodynamics of irreversible processes (De Groot \& Mazur, 1984). Later in 1960, Toupin \& Truesdell (Toupin \& Truesdell, 1960) started the modern thermodynamics of continuous media, or continuum mechanics, today the most comprehensive thermodynamic theory. This theory uses a rigorous mathematical treatment, is extensively applied in computer modeling of various materials and eliminates the artificial separation between thermodynamics and chemical kinetics, allowing a more consistent approach to chemical processes.

In this chapter, a radical simplification of thermodynamics of continuous media is obtained by imposing the homogeneous restriction on the process, that is, all the extensive and intensive properties of the system are functions of time, but are not functions of space. Improved physical understanding of some of the fundamental concepts of thermodynamics, such as internal energy, enthalpy, entropy, and the Helmholtz and Gibbs energies is presented. Further, the temporal view is applied to the first and second laws of thermodynamics. The conservation of linear and angular momenta, together with the rigid body concept, stresses the union with mechanics for the first law. For the second law, intrinsic characteristics of the system are central for understanding dissipation in thermally homogeneous processes. Moreover, including the definitions for non equilibrium states, the basic intensive properties of temperature, pressure and chemical potential are re-discussed. 
This is accomplished without making use of statistical methods and by selecting a mathematically coherent, but simplified temporal theory.

\section{Some basic concepts}

\subsection{Continuous media and thermodynamic properties}

The concept of continuous medium is derived from mathematics. The set of real numbers is continuous, since between any two real numbers there is infinity of numbers and it will always be possible to find a real number between the pair of original numbers, no matter how close they are (Mase \& Mase, 1999). Similarly, the physical space occupied by a body is continuous, although the matter is not continuous, because it is made up of atoms, which are composed of even smaller particles. Clearly, a material body does not fill the space it occupies, because the space occupied by its mass is smaller than the space occupied by its volume. But, according to the continuity of matter assumption, any chemical homogeneous body can be divided into ever-smaller portions retaining all the chemical properties of the original body, so one can assume that bodies completely fill the space they occupy. Moreover, this approach provides a solid mathematical treatment on the behavior of the body, which is correctly described by continuous real functions of time (Bassi, 2005a; Nery \& Bassi, 2009a).

With continuity imposed on matter, the body is called a system and, obviously, the mass and the volume of any system occupy the same space. If the outside boundary of the system is impermeable to energy and matter, the system is considered isolated. Otherwise, the system will be considered closed if the boundary that separates it from the outside is impermeable to mass only. The amount of any thermodynamic quantity is indirectly or directly perceived by an observer located within the system. A thermodynamic quantity whose amount cannot be verified by an observer located within an isolated system is not a property. The value assigned to any property is relative to some well established referential ( $\mathrm{m}$, mole etc.), but a referential does not need to be numerically well defined (the concept of mole is well established, but it is not numerically well defined).

Properties are further classified into intensive, additive extensive and non-additive extensive properties. Intensive properties are those that, at time $t$, may present real values at each point $\left\langle n_{1}, n_{2}, n_{3}>\right.$ of the system. Thus, if $a$ is an intensive property, there is a specific temporal function $a=a\left(t, n_{1}, n_{2}, n_{3}\right)$ defining the values of $a$. Examples of intensive properties are pressure, density, concentration, temperature and their inverses. In turn, extensive properties are those that have null value only (additive) or cannot present a real value (non-additive) at all points of the system. Examples of additive extensive properties are volume, mass, internal energy, Helmholtz and Gibbs energies, entropy and amount of substance. Inverses of additive extensive properties are non-additive extensive properties, but the most useful of these are products of additive extensive properties by inverses of additive extensive properties, such as the mean density of a system (Bassi, 2006a).

\subsection{Mathematical formalism}

Let a continuous function $y=f(x)$ be defined in an open interval of real numbers $(a, b)$. If a fixed real number $x$ within this range is chosen, there is a quotient,

$$
\frac{f(x+h)-f(x)}{h},
$$


where $h$ is a positive or negative real and $x+h$ is a real within the interval $(a, b)$. If $h$ approaches zero and the limit of the quotient tends to some well defined real value, then that limit defines the derivative of the function $y=f(x)$ at $x$ (Apostol, 1967),

$$
f^{\prime}(x)=\frac{\mathrm{d} y}{\mathrm{~d} x}=\lim _{h \rightarrow 0} \frac{f(x+h)-f(x)}{h} .
$$

The first equality of Equation 2 could still be represented by $\mathrm{d} y=f^{\prime}(x) \mathrm{d} x$, but not by multiplication of both its sides by the inverse of $d y$, because the values of $d y$ and $d x$ may be null and their inverses may diverge, thus the integrity of Equation 2 would not be maintained. It is fundamental to remember that the $\mathrm{d} y$ and $\mathrm{d} x$ values include not only finite quantities but necessarily zero, because there is a qualitative difference between null and finite quantities, no matter how small the finite quantities become. Thus, as well as Equation 2 cannot be multiplied by the inverse of $\mathrm{d} y$, the equation $\mathrm{d} y=f^{\prime}(x) \mathrm{d} x$ does not refer to an interval $y_{2}-y_{1}=f\left(x_{2}\right)-f\left(x_{1}\right)$, no matter how small the finite interval becomes, but uniquely to the fixed real value $x$ (as well as Equation 2).

Certainly, both the mathematical function and its derivative should maintain consistency with physical reality. For example, the $\mathrm{w}=\mathrm{w}(t)$ and $\mathrm{q}=\mathrm{q}(t)$ functions and their derivatives should express the intrinsic characteristics of work and heat and should retain their characteristics for any theory where these quantities are defined. Thus, because the Fourier equation for heat conduction defines $\frac{\mathrm{dq}}{\mathrm{d} t}$, acceptance of its validity implies accepting the existence of a differentiable temporal function $q=q(t)$ in any natural science. However, evidently the acceptance of the Fourier equation do not force all existing theories to include the equality $\mathrm{q}=\mathrm{q}(t)$. Surely, it will not be considered by timeless thermodynamics, but that is a constraint imposed on this theory.

Differential equations mathematically relate different quantities that an observer would be able to measure in the system. Some of these relations arise from specific properties of the material (constitutive functions), while others follow the physical laws that are independent of the nature of the material (thermodynamic functions). If the process is not specified, the differentiable function of state $z=u(x, y)$, and the process functions $z$, respectively correspond to an exact and inexact differential equations. Indeed, one has

$$
M(x, y) \mathrm{d} x+N(x, y) \mathrm{d} y=\mathrm{d} z,
$$

where $\frac{\partial M(x, y)}{\partial y}=\frac{\partial}{\partial y}\left(\frac{\partial u(x, y)}{\partial x}\right)$ and $\frac{\partial N(x, y)}{\partial x}=\frac{\partial}{\partial x}\left(\frac{\partial u(x, y)}{\partial y}\right)$ for $z=u(x, y)$. Because $\frac{\partial^{2} u(x, y)}{\partial y \partial x}=\frac{\partial^{2} u(x, y)}{\partial x \partial y}$, if

$$
\frac{\partial N(x, y)}{\partial x}=\frac{\partial M(x, y)}{\partial y}
$$

then $z=u(x, y)$ and the differential equation (Equation 3 ) is said to be exact. Otherwise, it is inexact. Thus, for an exact differential equation the function $z=u(x, y)$ can be found, but for 
solving an inexact differential equation the process must be specified. An important mathematical corollary indicates that the integral of an exact differential equation is independent of the path that leads from state 1 to state 2 (Bassi, 2005b; Agarwal \& O'Regan, 2008), because it equals $z_{2}-z_{1}=u\left(x_{2}, y_{2}\right)-u\left(x_{1}, y_{1}\right)$, while this is not true for integrals of inexact differential equations.

Mathematically, the concept of state comprises the smallest set of measurements of system properties, at time $t$, enough to ensure that all measures of properties of the system are known, at that very moment. The definition of state implies that if $\mathrm{X}$ is the value of any property of the system at instant $t$ and $\Xi$ is the state of the system at that same time, there must be a constitutive or thermodynamic function $X=X(\Xi)$. On the other hand, if $Y$ does not correspond to the value of a property of the system at time $t$, the existence of a function $Y=Y(\Xi)$ is not guaranteed. This shows that all integrals of exact differential equations are function of state differences between two states, while differential equations involving the differentials of properties included in $\Xi$ generally are inexact (Nery \& Bassi, 2009b). Thus, all properties are functions of state and, if the process is not specified, all functions of state are properties.

\subsection{Relative and absolute scales}

Consider a sequence of systems ordered according to the continuous increment of a specific property of them, as for example their volume. This ordering may be represented by a continuous sequence of real numbers named a dimensionless scale. Dimensionless scales can be related each other by choosing functions whose derivatives are always positive. Linear functions do not alter the physical content of the chosen property, but non-linear ones do not expand or contract proportionally all scale intervals. Thus, dimensionless scales related by non-linear functions attribute different physical characteristics to the considered property. For instance, because the dimensionless scales corresponding to empirical and absolute temperatures are related by a non-linear function, empirical temperatures cannot be substituted for absolute temperatures in thermodynamic equations.

The entire real axis is a possible dimensionless scale. Because the real axis does not have a real number as a lower bound neither as an upper bound, it is not sufficient to choose a value in the scale and relate this value to a particular system, in order to convert the dimensionless scale to a dimensional one (Truesdell, 1984). To do this, it is essential to employ at least two values, as for empirical temperature scales. But only one value is needed if a pre-defined unit is used, as in the case of the Pascal unit for pressure $\left(\mathrm{Pa}=\mathrm{Kg} \mathrm{m}^{-1} \mathrm{~s}^{-2}\right.$, where $\mathrm{Kg}$, $\mathrm{m}$ and $\mathrm{s}$ are, respectively, the pre-defined units for mass, distance and time). The dimensional scales for empirical temperatures and for pressure are examples of relative scales.

So, if $\mathrm{X}$ belongs to the real axis, for $-\infty<\mathrm{X}<\infty$ one may propose the new dimensionless scale

$$
\mathrm{Y}=\exp (\mathrm{X}) \text {. }
$$

This new scale, contrasting with the previous one, only includes the positive semi-axis of real numbers with the zero lower bound being as unattainable as the lower bound of the real axis, $-\infty$. By imposing $X=0$, Equation 5 gives $Y=1$, where the dimensionless 1 can be related to any system for defining the scale unit. Any scale containing only the positive semi-axis of real numbers that assigns a well defined physical meaning to $Y=1$ is a 
dimensional scale called absolute. The physical contents of some properties, as for example the volume, require absolute scales for measuring their amounts in the system (for the volume, $\mathrm{Y}=1$ may be assigned to $1 \mathrm{~m}^{3}$ and there is not a null volume system).

\section{First law of thermodynamics}

\subsection{Internal energy}

According to the thermodynamics of continuous media, the mathematical expression for the first law of thermodynamics is a balance of energy that, along with the balance equations of mass and linear and angular momenta, applies to phenomena that involve the production or absorption of heat. In this approach, conservation of linear and angular momenta is explicit in the energy balance, while in classical thermodynamics conservation of linear and angular momenta are implicitly assumed. Actually, because classical thermodynamics focuses its attention on systems which are macroscopically stationary, linear and angular momenta are arbitrarily zero, restricting the study of several physical systems (Liu, 2002).

The principle of conservation of energy was first enunciated by Joule, near the mid of XIX century, who demonstrated through numerous experiments that heat and work are uniformly and universally inter-convertible. Moreover, the principle of conservation of energy requires that for any positive change in the energy content of the system, there must be an inflow of energy of equal value. Similarly, for any negative change of the energy content of the system, there must be liberation of the same energy value.

Consider a body whose composition is fixed. Moreover, suppose that the positions and relative orientations of the constituent particles of the body are unchanged, but the body can move in space. This body is defined as rigid body and its energy content is the body's energy $E_{R}$. Now, consider that the restrictions on the number of particles, their positions and orientations are abolished, so the body's energy is E. Thus, the energy content of the body can be separated into two additive parts

$$
\mathrm{E}=U+\mathrm{E}_{\mathrm{R}},
$$

where $U$ is the internal energy and represents the sum of the energies of the motions, of the constituent particles and into them, which do not change the total linear and angular momenta of the body (internal motions).

While the energy of the rigid body is well defined by the laws of mechanics, the comprehension of internal energy values depends on the microscopic model adopted to describe material bodies. The difference $\Delta U_{a \rightarrow b}=U\left(t_{b}\right)-U\left(t_{a}\right)$, between the internal energy at two instants $t_{a}$ and $t_{b}$ of a gas supposed ideal, can be experimentally determined. However, it is not possible to experimentally determine the internal energy of any body at instant $t$.

Similarly, the energy exchange between a body and its exterior is divided into two additive portions named heat and work. Heat, $q$, is an exchange of energy in which total linear and angular momenta of the body, as well as total linear and angular momenta of its exterior, are not changed. Thus, heat involves only the internal energies of the body and its exterior and cannot be absorbed or emitted by the energy of a rigid body (Moreira \& Bassi, 2001; Bassi, 2006b). In turn, work, w, involves both the internal and rigid body energies. Hence, there is no restriction on the rigid body absorption or emission of work (Williams, 1971). Equation 6, as well as the concepts of rigid body energy, internal energy, heat and work is valid not only for bodies, but also for systems. 
Considering the time of existence of a process in a closed system, the heat exchanged from the initial instant $t_{\#}$ until the instant $t$ is denoted by $\Delta \mathrm{q}(t)=\mathrm{q}(t)-\mathrm{q}_{\#}$, where $\mathrm{q}_{\#}$ represents the heat exchanged from a referential moment until the initial instant $t_{\#}$ of the process and $\mathrm{q}(t)$ indicates the heat exchanged from the referential moment until instant $t$. Likewise, one has $\Delta \mathrm{w}(t)=\mathrm{w}(t)-\mathrm{w}_{\#}, \Delta \mathrm{w}_{\mathrm{R}}(t)=\mathrm{w}_{\mathrm{R}}(t)-\mathrm{w}_{\mathrm{R} \#}$ and, by imposing $\mathrm{q}_{\#}=0, \mathrm{w}_{\#}=0$ and $\mathrm{w}_{\mathrm{R} \#}=0$, respectively

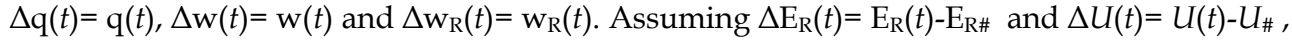
energy conservation implies that

$$
\Delta \mathrm{E}_{R}(t)+\Delta U(t)=\Delta \mathrm{q}(t)+\Delta \mathrm{w}_{\mathrm{R}}(t)+\Delta \mathrm{w}(t),
$$

where $\Delta \mathrm{w}(t)$ indicates the portion of the work that is transformed into internal energy or comes from it.

The more general statement of the first law of thermodynamics is:

“The internal energy and the energy of rigid body do not interconvert (Šilhavý, 1989)."

Therefore, according to the statement on the first law and Equation 7,

$$
\Delta \mathrm{E}_{\mathrm{R}}(t)=\Delta \mathrm{w}_{\mathrm{R}}(t),
$$

and, subtracting Equation 8 from Equation 7,

$$
\Delta U(t)=\Delta \mathrm{q}(t)+\Delta \mathrm{w}(t) .
$$

Equation 9 is the mathematical expression of the first law of thermodynamics for closed systems. For the range from $t_{a}$ to $t_{b}$, where $t_{\#}<t_{a} \leq t \leq t_{b}<t^{\#}$, Equation 9 may be written

$$
\int_{t_{a}}^{t_{b}} \frac{\mathrm{d} U}{\mathrm{~d} t} \mathrm{~d} t=\int_{t_{a}}^{t_{b}} \frac{\mathrm{dq}}{\mathrm{d} t} \mathrm{~d} t+\int_{t_{a}}^{t_{b}} \frac{\mathrm{d} w}{\mathrm{~d} t} \mathrm{~d} t,
$$

and, by making $t_{a} \rightarrow t$ and $t_{b} \rightarrow t$, the limit of Equation 10 is

$$
\frac{\mathrm{d} U}{\mathrm{~d} t}=\frac{\mathrm{dq}}{\mathrm{d} t}+\frac{\mathrm{dw}}{\mathrm{d} t}
$$

where $\frac{\mathrm{d} U}{\mathrm{~d} t}$ is the rate of change of internal energy of the system at time $t$, and $\frac{\mathrm{dq}}{\mathrm{d} t}$ and $\frac{\mathrm{dw}}{\mathrm{d} t}$ are respectively the thermal and the non thermal powers that the system exchanges with the outside at that instant. Defining the differentials

$$
\mathrm{d} U=\frac{\mathrm{d} U}{\mathrm{~d} t} \mathrm{~d} t, \mathrm{dq}=\frac{\mathrm{dq}}{\mathrm{d} t} \mathrm{~d} t \text { and } \mathrm{dw}=\frac{\mathrm{dw}}{\mathrm{d} t} \mathrm{~d} t,
$$

Equation 11 may be written

$$
\mathrm{d} U=\mathrm{dq}+\mathrm{dw} .
$$

Considering the entire range of existence of a process $t_{\#}<t<t^{\#}$ and imposing $\mathrm{q}_{\#}=0$ and $\mathrm{w}_{\#}=0$, Equation 9 can be rewritten

$$
\Delta U=\mathrm{q}+\mathrm{w},
$$


which is the most usual form of the first law. Equations 9 to 14 reflect the conservation of energy in the absence of changes of total linear and angular momenta.

Because differentials are not extremely small finite intervals, it should be noted that Equation 9 cannot be extrapolated to Equation 13. But in some textbooks Equation 13 is proposed considering that: (a) $\mathrm{d} U$ is an exact differential, but $\mathrm{dq}$ and $\mathrm{dw}$ are inexact differentials or (b) $\mathrm{dq}$ and $\mathrm{dw}$ are finite intervals, while $\mathrm{d} U$ is a differential. Such considerations arise from a mistaken view of the differential concept. Indeed: (1) in order to a differential equation to have mathematical meaning, its differentials must be defined using derivatives, as in Equations 3 (by using the process specifications if needed) and 12; (2) the subtraction of two different well-defined real values corresponds to a well-defined finite interval and produces a well-defined real, no matter how small, but never a differential, which is an undetermined real and (3) there are exact and inexact differential equations, but there is not such classification for differentials. In short, Equation 13 is a consequence of Equation 9 if and only if the differentials $\mathrm{d} U$, $\mathrm{dq}$ and $\mathrm{dw}$ are defined using derivatives, while the validity of Equation 14 does not require this (Gurtin, 1971; Nery \& Bassi, 2009b).

\subsection{Enthalpy}

Suppose a closed system whose outside homogeneously exerts, on the system boundary, a well defined constant pressure $\mathrm{p}^{\prime}$ during the entire existence of a process occurring in the system, including the initial and final instants of the process. Additionally, consider

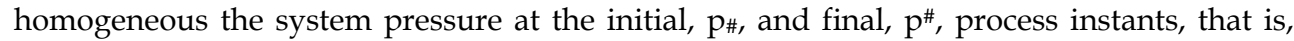
consider that, at the initial and final process instants, the system is in mechanical equilibrium with outside, so that $\mathrm{p}_{\#}=\mathrm{p}^{\#=} \mathrm{p}^{\prime}$. Therefore, for a process under constant pressure it is necessary that the system be in mechanical equilibrium at $t_{\#}$ and $t^{\#}$, but it is not necessary that this also occurs during the existence interval of the process, $t_{\#}<t<t^{\#}$. If, excluding the volumetric work performed by $\mathrm{p}^{\prime}$ or against $\mathrm{p}^{\prime}, \Delta \mathrm{w}_{\mathrm{nv}}(t)$ is the work exchanged by the system from the initial instant to an instant $t$ such that $t_{\#}<t<t^{\#}$, thus, for a process under constant pressure occurring in a closed system,

$$
\Delta \mathrm{wnv}_{\mathrm{nv}}(t)+\Delta \mathrm{q}(t)=\Delta U(t)+\Delta(\mathrm{pV})(t)=\Delta \mathrm{H}(t)
$$

because the enthalpy at instant $t, \mathrm{H}(t)$, is defined by

$$
\mathrm{H}(t)=U(t)+(\mathrm{pV})(t)
$$

If $\Delta \mathrm{wnv}_{\mathrm{nv}}(t)=0$, Equation 15 indicates that the heat exchanged with the outside during a process under constant pressure is the enthalpy change $\Delta \mathrm{H}(t)$ (Planck, 1945). This result is of fundamental importance for thermo-chemistry, because in this system the enthalpy behaves similarly to the internal energy in a closed system limited by rigid walls. In analogy to the mathematical expression of the first law of thermodynamics for closed systems (Equation 9), $\Delta \mathrm{H}$ indicates the module and the direction of the exchange of energy $\Delta \mathrm{w}_{\mathrm{nv}}(t)+\Delta \mathrm{q}(t)$ between the system and its surroundings. Considering $\Delta \mathrm{w}_{\mathrm{nv}}(t)=0$, if $\Delta \mathrm{H}<0$ the process is said to be exothermic and, if $\Delta \mathrm{H}>0$, the process is endothermic.

\section{Second law of thermodynamics}

\subsection{Statement for the second law}

The first law of thermodynamics is not sufficient to determine the occurrence of physical or chemical processes. Whereas the first law addresses just the energetic content of system, the 
second law demands further conditions for the existence of a process. Treatises on classical thermodynamics contain several statements about the second law, which are frequently associated with the works of Clausius, Kelvin, Carnot and Planck. Despite some differences among the various statements, all of them claim that to produce an amount of work in a cyclic process, the system must not only absorb heat, but it must also emit some amount of it (Kestin, 1976).

This is equivalent to the establishment that, for any closed system at a homogeneous temperature, work and internal energy may always be converted into heat according to the first law, but there is a limit for the rate of absorbing heat,

$$
\frac{\mathrm{dq}}{\mathrm{d} t} \leq \mathrm{T} \frac{\mathrm{dS}}{\mathrm{d} t}
$$

and for the rate of producing work (Šilhavý, 1997),

$$
-\frac{\mathrm{dw}}{\mathrm{d} t} \leq \mathrm{T} \frac{\mathrm{d} S}{\mathrm{~d} t}-\frac{\mathrm{d} U}{\mathrm{~d} t}
$$

where $\mathrm{T}$ is the homogeneous absolute temperature and $\mathrm{S}$ is the entropy. The variables $\mathrm{T}, \mathrm{S}$ and $U$ correspond to properties of the closed system but, because time derivatives of state functions are not state functions (Nery \& Bassi, 2009b), Equation 17 does not necessarily impose a constraint on the rate of heat absorption. On the contrary, given this rate, Equation 17 causes an entropy rate increase at least equal to $\frac{1}{\mathrm{~T}} \frac{\mathrm{dq}}{\mathrm{d} t}$ and, given $\frac{\mathrm{d} U}{\mathrm{~d} t}$, Equation 18 shows that a larger entropy rate increase corresponds to a larger rate of producing work. But the system must return to the same state for cyclic processes, thus in such processes restrictions are imposed to the variations of state functions. Indeed, Equation 17 indicates that, for thermally homogeneous cyclic processes that occur in closed systems (Serrin, 1979),

$$
\oint \frac{\mathrm{dq}}{\mathrm{T}} \leq 0 .
$$

Equation 17 also introduces the idea of dissipation (Šilhavý, 1983). If the dissipation is defined by

$$
\frac{\mathrm{d} \delta}{\mathrm{d} t} \equiv \mathrm{T} \frac{\mathrm{dS}}{\mathrm{d} t}-\frac{\mathrm{dq}}{\mathrm{d} t}
$$

Equation 17 shows that

$$
\frac{\mathrm{d} \delta}{\mathrm{d} t} \geq 0
$$

Hence, the second law of thermodynamics asserts that there exists in nature an amount which, by changes on a closed system at homogeneous temperature, either remains constant (non-dissipative processes) or increases (dissipative processes). The concept of dissipation presented here is analogous to friction (Truesdell, 1984). However, it is an internal friction in 
the system and not between the system and its outside. Dissipation always occurs when, in the state considered, there is a tendency to change the internal motions of the system.

So far, the second law of thermodynamics has been defined for thermally homogeneous closed systems. If additional restrictions are imposed on the system such as system isolation, according to Equations 17 and 21 the second law states that

$$
\frac{\mathrm{d} S}{\mathrm{~d} t} \geq 0 \text { and } \frac{\mathrm{d} \delta}{\mathrm{d} t} \geq 0
$$

Equation 22 confirms that dissipation may occur even for a system which do not exchange energy with its outside, reinforcing the fact that dissipation is an internal phenomenon of the system. Isolated systems are not the only special thermally homogeneous closed systems of interest. Thus, some specific situations are detailed in the following text. But, first, note that thermodynamic reservoirs are not considered in this approach because, by definition, reservoirs are systems which do not obey the same physical laws of the system under study. However, it is possible to make experimentally confirmed deductions by imposing that the environment obeys the same physical laws as the body (Hutter, 1977; Serrin, 1979; Nery \& Bassi, 2009b). Now, consider a thermally homogeneous closed system under:

Adiabatic process: If no heat exchange with the outside is imposed, $\frac{\mathrm{dq}}{\mathrm{d} t}=0$ and, according to Equation 17,

$$
\frac{\mathrm{d} S}{\mathrm{~d} t} \geq 0
$$

An important consequence obtained from Equation 23 is that, in a non-dissipative process, the words "adiabatic" and "isentropic" have the same meaning, but for a dissipative adiabatic process clearly $\frac{\mathrm{dS}}{\mathrm{d} t}>0$ (Truesdell, 1991).

Isoenergetic process: If an isoenergetic process is considered, but interactions between the system and its outside are allowed, Equation 18 shows that

$$
-\frac{\mathrm{dw}}{\mathrm{d} t} \leq \mathrm{T} \frac{\mathrm{dS}}{\mathrm{d} t} .
$$

Thus, for a non-dissipative process the entropy of a system can be decreased by doing work on the system. This is a very interesting assertion, because it eliminates the wrong idea that in any process whatever the entropy of a system always remains either constant or increases. Obviously, if the absence of both volumetric and non-volumetric work is imposed, changes of $\frac{\mathrm{d} S}{\mathrm{~d} t}$ coincide with those of an adiabatic process (Day, 1987).

Isentropic process: If entropy $\mathrm{S}$ is maintained constant, from Equation 17

$$
\frac{\mathrm{dq}}{\mathrm{d} t} \leq 0
$$


Thus, heat cannot be absorbed in an isentropic process. A non-dissipative isentropic process is adiabatic and the change of internal energy coincides with the work exchanged. In the absence of both volumetric and non-volumetric work, Equation 25 becomes

$$
\frac{\mathrm{d} U}{\mathrm{~d} t} \leq 0
$$

Thus, if no work is exchanged during the process, the internal energy does not increase in an isentropic process.

Isothermal process: If the homogeneous temperature is kept constant in time, the time derivative of the Helmholtz energy,

$$
\mathrm{A}(t)=U(t)-(\mathrm{TS})(t)
$$

is

$$
\frac{\mathrm{dA}}{\mathrm{d} t}=\frac{\mathrm{d} U}{\mathrm{~d} t}-\mathrm{T} \frac{\mathrm{d} S}{\mathrm{~d} t}
$$

and, using Equation 28, Equation 18 can be described by

$$
\frac{\mathrm{dA}}{\mathrm{d} t} \leq \frac{\mathrm{dw}}{\mathrm{d} t}
$$

Hence, in an isothermal process, the increase of Helmholtz energy is not greater than the work done on the system. In addition, if no work is exchanged during the process,

$$
\frac{\mathrm{dA}}{\mathrm{d} t} \leq 0
$$

Equation 30 implies that the Helmholtz energy does not increase. Note that all these conclusions are restricted to isothermal processes. If the process is thermally homogeneous, but not isothermal, instead of Equation 29 the correct relation is $\frac{\mathrm{dA}}{\mathrm{d} t} \leq \frac{\mathrm{dw}}{\mathrm{d} t}-\mathrm{S} \frac{\mathrm{dT}}{\mathrm{d} t}$, which is far more complicated.

Isothermal-isobaric process: If both temperature and pressure are homogeneous and constant in time, the time derivative of the Gibbs energy,

$$
\mathrm{G}(t)=\mathrm{H}(t)-(\mathrm{TS})(t)
$$

is

$$
\frac{\mathrm{dG}}{\mathrm{d} t}=\frac{\mathrm{dH}}{\mathrm{d} t}-\mathrm{T} \frac{\mathrm{dS}}{\mathrm{d} t}
$$

and Equation 18 can be replaced by

$$
\frac{\mathrm{d} U}{\mathrm{~d} t}-\mathrm{T} \frac{\mathrm{dS}}{\mathrm{d} t}+\mathrm{p} \frac{\mathrm{dV}}{\mathrm{d} t} \leq \frac{\mathrm{dw} \mathrm{nv}_{\mathrm{n}}}{\mathrm{d} t}
$$

Using Equation 16, Equation 33 may be written 


$$
\frac{\mathrm{dH}}{\mathrm{d} t}-\mathrm{T} \frac{\mathrm{dS}}{\mathrm{d} t} \leq \frac{\mathrm{dw} \mathrm{wv}_{\mathrm{nv}}}{\mathrm{d} t}
$$

or, using Equation 32,

$$
\frac{\mathrm{dG}}{\mathrm{d} t} \leq \frac{\mathrm{dw}_{\mathrm{nv}}}{\mathrm{d} t}
$$

For example, for a spontaneous process $\frac{\mathrm{dw}_{\mathrm{nv}}}{\mathrm{d} t}$ may be zero, thus $\frac{\mathrm{dG}}{\mathrm{d} t} \leq 0$. If an isothermalisobaric endothermic reaction is spontaneous, $\mathrm{T} \frac{\mathrm{dS}}{\mathrm{d} t}$ is positive and large enough to surpass the positive value $\frac{\mathrm{dH}}{\mathrm{d} t}$. Therefore, isothermal-isobaric endothermic reactions are driven by the increase of entropy. On the other hand, for a spontaneous isothermal-isobaric exothermic reaction, entropy may decrease but $\frac{\mathrm{dH}}{\mathrm{d} t}$ must be negative enough to surpass the negative value $\mathrm{T} \frac{\mathrm{d} S}{\mathrm{~d} t}$.

Although thermally homogeneous processes must be studied, natural (heterogeneous) processes must also be mentioned. All natural processes will approach thermal homogeneity as the forward process rate decreases, in relation to a finite thermal homogenization rate considered constant. If this happens in a closed system, the process will approach obedience to Equation 17. Nevertheless, because the process tends to a stationary state, the dissipation $\frac{\mathrm{d} \delta}{\mathrm{d} t}$ tends to zero faster than Equation 17 becomes obeyed. This means that when the forward process rate of the process tends to zero, both a thermally homogeneous dissipative process and a natural process tend towards a thermally homogeneous non-dissipative process. On the other hand, a natural process will approach a thermally homogeneous dissipative process when its thermal homogenization rate is increased, in relation to a finite and constant forward rate of the natural process. Thus, Equation 17 is a limiting equation for natural processes.

\subsection{Maximization of missing information}

A possible statistical way for expressing the second law of thermodynamics is:

"A system may change over time until the state with the highest density of possible microstates is reached. Once this state is achieved, the system cannot alter it anymore, unless the conditions imposed on the system are modified."

To illustrate this statement of the second law, consider a sphere divided by an imaginary diametrical plane into two compartments I and II. Also, consider two indistinguishable mathematical points moving at random, so the probability of occurrence of any of the following microstates is equal to $1 / 4$ : " $x$ in $\mathrm{I}, y$ in $\mathrm{II}^{\prime \prime}, " y$ in $\mathrm{I}, x$ in $\mathrm{II}^{\prime \prime}$, " $x$ and $y$ in $\mathrm{I}^{\prime \prime}$ and " $x$ and $y$ in II". However, since the $x$ and $y$ points are indistinguishable, the probability of the state "one point in I, one point in II" is twice the probability of occurrence for each one of the states "two points in I" and "two points in II" (Bassi, 2005c). Because probability theory rests upon set theory, it is reasonable to introduce states as sets of equally probable microstates. 
Now, suppose a gas consisting of only 10 molecules occupying the entire volume of a closed vessel. The probability that all molecules are in the left half of the vessel at the same time $t$ is $1 / 2^{10}=1 / 1024$, that is, for every 1024 seconds this configuration could be observed, on average, during one second. However, thermodynamics deals only with macroscopic systems, where the number of constituents is of the order of the Avogadro constant. So, for one mole of molecules in a gaseous state, the probability that all they are in the left or right half of the vessel is, for all purposes, zero and then one can consider that such state does not exist. But, because the thermodynamic state varies continuously, the concept of the number of microstates corresponding to each state must be replaced by the continuously varying non-dimensional density of microstates, $\gamma \geq 1$, related to each state (Fermi, 1956).

In general, for a macroscopic system the density of possible microstates may be considered null for all states, except for the state with the highest density of possible microstates, which is called the stable state. But, because potential barriers can restrain changes of state, the system may remain in an unstable state until a perturbation suddenly alters the system state. This is the reason for not imposing that the system will change over time until the state with the highest density of possible microstates is reached, in the statistical statement of the second law. Note that, as the density of possible microstates corresponding to the state increases, the partial knowledge about the state of the system decreases. Thus, in the stable state the ignorance (missing information) about the characteristics of the system is maximized.

\subsection{Missing structural information and other missing information}

In the previous section 4.1 the existence of a thermodynamic property called entropy was introduced, which helps in understanding how a thermodynamic process will evolve. In the present section, an interpretation of entropy is presented, based on the structural characteristics of the system. First, by supposing that the values for all properties that cannot change in an isolated system (such as mass, volume, and internal energy) are already known, for any system define structural information as additional information. Then, for any system, entropy is proportional to the quantity $\varphi$ of missing structural information (Brillouin, 1962; Gray, 1990).

In an isolated system the missing structural information is associated with the density of microstates by

$$
\varphi=\ln (\gamma),
$$

where $\mathrm{c}$ is an arbitrary constant of proportionality that defines the unit for measurement of missing structural information. By considering $\mathrm{c}=k_{\mathrm{B}}$, where $k_{\mathrm{B}}$ is the Boltzmann constant, Equation 36 is written

$$
\mathrm{S}=k_{\mathrm{B}} \ln (\gamma),
$$

which is the familiar relationship between entropy and the density of microstates of the isolated system (Boltzmann, 1964). Note that the entropy is proportional to the missing structural information for whatever system but, only for an isolated system, entropy is proportional to the logarithm of the density of microstates. Using the statistical statement for the second law, Equation 37 indicates that:

"In an isolated system, the entropy never decreases as time increases." 
Therefore, the combined effect of the first and second laws of thermodynamics states that, as time progresses, the internal energy of an isolated system may redistribute without altering its total amount, in order to increase the entropy until the latter reaches a maximum, at the stable state. This statement coincides with the known extreme principles (Šilhavý, 1997).

The interpretation of entropy as a measure of the well defined missing structural information allows a more precise comprehension of this important property, without employing subjective adjectives such as organized and unorganized. For example, consider a gaseous isolated system consisting of one mole of molecules and suppose that all the molecules occupy the left or right half of the vessel. The entropy of this state is lower than the entropy of the stable state because, for an isolated system, the entropy is related to the density of microstates (which, for this state, is lower than the density for the stable state) and, for any system, the entropy is related to the ignorance about the structural conditions of the system (which, for this state, is lower than the ignorance for the stable state). Thus, the entropy does not furnish any information about whether this state is ordered or not (Michaelides, 2008).

Because $\gamma \geq 1$, according to Equation 37 entropy is an additive extensive property whose maximum lower bound value is zero, so that $S \geq 0$. But it is not assured that, for all systems, $\mathrm{S}$ can in fact be zero or very close zero. For instance, unlike crystals in which each atom has a fixed mean position in time, in glassy states the positions of the atoms do not cyclically vary. That is, even if the temperature should go to absolute zero, the entropies of glassy systems would not disappear completely, so that they present the residual entropy

$$
\mathrm{S}_{\mathrm{RES}}=k_{\mathrm{B}} \ln \left(\gamma_{\mathrm{G}}\right) \text {, }
$$

where $\gamma_{\mathrm{G}}>1$ represents the density of microstates at $0 \mathrm{~K}$. This result does not contradict Nernst's heat theorem. Indeed, in 1905 Walther Nernst stated that the variation of entropy for any chemical or physical transformation will tend to zero as the temperature approaches indefinitely absolute zero, that is,

$$
\lim _{\mathrm{T} \rightarrow 0}(\Delta S)=0 .
$$

But there is no doubt that the value of $S_{\text {RES }}$, for any substance, is negligible when compared with the entropy value of the same substance at $298.15 \mathrm{~K}$. Therefore, at absolute zero the entropy is considered to be zero. This assertion is equivalent to the statement made by Planck in 1910 that, as the temperature decreases indefinitely, the entropy of a chemical homogeneous body of finite density tends to zero (Planck, 1945), that is,

$$
\lim _{\mathrm{T} \rightarrow 0}(\mathrm{~S})=0 \text {. }
$$

This assertion allows the establishment of a criterion to distinguish stable states from steady states, because stable states are characterized by a null limiting entropy, whereas for steady states the limiting entropy is not null (Šilhavý, 1997).

Although it is known that $\gamma$ is not directly associated with the entropy for a non-isolated system, $r$ still exists and is related to some additive extensive property of the system denoted by $\zeta$ (Tolman, 1938; Mcquarrie, 2000). By requiring that the unit for $\zeta$ is the same as for $S$, the generalized Boltzmann equation is written 


$$
\zeta=k_{\mathrm{B}} \ln (\gamma),
$$

where $\zeta$ is proportional to some kind of missing information. Considering the special processes discussed in the previous section 4.1, in some cases the property denoted by $\zeta$ (Equation 41) can be easily found. For instance, since $\frac{\mathrm{dA}}{\mathrm{d} t} \leq 0$ for an isothermal process in a closed system which does not exchange work with its surroundings, then $\zeta=-\frac{A}{T}=S-\frac{U}{T}$ for thermally homogeneous closed systems that cannot exchange work with the outside. Analogously, if both the temperature and the pressure of a closed system are homogeneous and the system can only exchange volumetric work with the outside, then $\zeta=-\frac{G}{T}=S-\frac{H}{T}$.

\section{Homogeneous processes}

\subsection{Fundamental equation for homogeneous processes}

During the time of existence of a homogeneous process, the value of each one of the intensive properties of the system may vary over time, but at any moment the value is the same for all geometric points of the system. The state of a homogeneous system consisting of $J$ chemical species is characterized by the values of entropy, volume and amount of substance for each one of the $J$ chemical species, that is, the state is specified by the set of values $\Phi=\left\langle\mathrm{S}, \mathrm{V}, \mathrm{n}_{1}, \ldots, \mathrm{n}_{\jmath}\right\rangle$. Obviously, this assertion implies that all other independent properties of the system, as for instance its electric or magnetic polarization, are considered material characteristics which are held constant during the time of existence of the process. Should some of them vary, the set of values $\Phi$ would not be enough for specifying the state of the system, but such variations are not allowed in the usual theory. This assertion also implies that $S$ exists, independently of satisfying the equality $\mathrm{dq}=\mathrm{TdS}$. This approach was proposed by Planck and is very important, since it allows introducing the entropy without employing concepts such as Carnot cycles (Planck, 1945).

Thus, at every moment $t$ the value of the internal energy $U$ is a state function $U(t)=U(S(t)$, $\left.\mathrm{V}(t), \mathrm{n}_{1}(t), \ldots, \mathrm{n}_{J}(t)\right)$. Moreover, since this function is differentiable for any set of values $\Phi=<\mathrm{S}$, $\mathrm{V}, \mathrm{n}_{1}, \ldots, \mathrm{n}_{>}>$, the equation defining the relationship between $\mathrm{d} U, \mathrm{dS}, \mathrm{dV}$, and $\mathrm{dn}_{1}, \ldots, \mathrm{dn}_{J}$, is the exact differential equation

$$
\mathrm{d} U=\frac{\partial U}{\partial S}(\Phi) \mathrm{d} S+\frac{\partial U}{\partial \mathrm{V}}(\Phi) \mathrm{dV}+\sum_{j=1}^{J} \frac{\partial U}{\partial \mathrm{n}_{\mathrm{j}}}(\Phi) \mathrm{dn}_{\mathrm{j}} .
$$

The internal energy, the entropy, the volume and the amounts of substance are called the phase (homogeneous system) primitive properties, that is, all other phase properties can be derived from them. For instance, the temperature, the pressure and the chemical potential of any chemical species are phase intensive properties respectively defined by $\mathrm{T}=\frac{\partial U}{\partial S}(\Phi)$, $\mathrm{p}=-\frac{\partial U}{\partial \mathrm{V}}(\Phi)$ and $\mu_{\mathrm{j}}=\frac{\partial U}{\partial \mathrm{n}_{\mathrm{j}}}(\Phi)$ for $j=1, \ldots, J$. Thus, by substituting $\mathrm{T}, \mathrm{p}$ and $\mu_{\mathrm{j}}$ for their corresponding derivatives in Equation 42, the fundamental equation of homogeneous processes is obtained, 


$$
\mathrm{d} U=\mathrm{TdS}-\mathrm{pdV}+\sum_{j=1}^{J} \mu_{\mathrm{j}} \mathrm{dn} \mathrm{j}_{\mathrm{j}} .
$$

Equation 43 cannot be deduced from both Equation 13 and the equalities $d q=T d S$ and $d w=-p d V$ (Nery \& Bassi, 2009b). Since the phase can exchange types of work other than the volumetric one, these obviously should be included in the expression of first law, but the fundamental equation of homogeneous processes might not be altered. For instance, an electrochemical cell exchanges electric work, while the electric charge of the cell does not change, thus it is not included in the variables defining the system state, and a piston expanding against a null external pressure produces no work, but the cylinder volume is not held constant, thus the volume is included in the variables defining the system state. Moreover, there is not a "chemical work", because chemical reactions may occur inside isolated systems, but work is a non-thermal energy exchanged with the system outside (section 3.1).

Equations 13 and 43 only coincide for non-dissipative homogeneous processes in closed systems that do not alter the system composition and exchange only volumetric work with the outside. But neither Equation 13, nor Equation 43 is restricted to non-dissipative processes, and a differential equation for dissipative processes cannot be inferred from a differential equation restricted to non-dissipative ones, because differential equations do not refer to intervals, but to unique values of the variables (section 2.2), so invalidating an argument often found in textbooks. Indeed, homogeneous processes in closed systems that do not alter the system composition and exchange only volumetric work with the outside cannot be dissipative processes. Moreover, Equation 13 is restricted to closed systems, while Equation 43 is not. In short, Equation 43 , as well as the corresponding equation in terms of time derivatives,

$$
\frac{\mathrm{d} U}{\mathrm{~d} t}=\mathrm{T} \frac{\mathrm{d} S}{\mathrm{~d} t}-\mathrm{p} \frac{\mathrm{dV}}{\mathrm{d} t}+\sum_{j=1}^{J} \mu_{\mathrm{j}} \frac{\mathrm{dn}_{\mathrm{j}}}{\mathrm{d} t},
$$

refer to a single instant and a single state of a homogeneous process, which needs not to be a stable state (a state in thermodynamic equilibrium).

The Equations 43 and 44 just demand that the state of the system presents thermal, baric and chemical homogeneity. Because each phase in a multi-phase system has its own characteristics (for instance, its own density), $\Phi$ separately describes the state of each phase in the system. But, because the internal energy, the entropy, the volume and the amounts of substance are additive extensive properties, their differentials for the multi-phase system can be obtained by adding the corresponding differentials for a finite number of phases. Thus, the thermal, baric and chemical homogeneities guarantee the validity of Equations 43 and 44 for multi-phase systems containing a finite number of phases.

Further, if an interior part of the system is separated from the remaining part by an imaginary boundary, this open subsystem will still be governed by Equations 43 and 44 . Because any additive extensive property will approach zero when the subsystem under study tends to a point, sometimes it is convenient to substitute $u=u\left(s, v, c_{1}, \ldots, c_{j}\right)$, where $\mathrm{u}=\frac{U}{\mathrm{M}}, \mathrm{s}=\frac{\mathrm{S}}{\mathrm{M}}, \mathrm{v}=\frac{\mathrm{V}}{\mathrm{M}}, \mathrm{cj}=\frac{\mathrm{n}_{\mathrm{j}}}{\mathrm{M}}$ for $j=1, \ldots, J$, and $\mathrm{M}$ is the subsystem mass at instant $t$, for $U=U\left(\mathrm{~S}, \mathrm{~V}, \mathrm{n}_{1}, \ldots, \mathrm{n}_{J}\right)$. Hence, the equation 


$$
\mathrm{du}=\mathrm{Tds}-\mathrm{pdv}+\sum_{j=1}^{J} \mu_{\mathrm{j}} \mathrm{dc}_{\mathrm{j}},
$$

may substitute Equation 43. Indeed, Equation 45 is a fundamental equation of continuum mechanics.

\subsection{Thermodynamic potentials}

Not only is the function $U=U(\Phi)$ differentiable for all values of the set $\Phi$, but also the functions $\frac{\partial U}{\partial S}(\Phi), \frac{\partial U}{\partial \mathrm{V}}(\Phi)$, and $\frac{\partial U}{\partial \mathrm{n}_{\mathrm{j}}}(\Phi)$ for $j=1, \ldots, J$ are differentiable. Moreover, because $\frac{\partial^{2} U}{\partial S^{2}}(\Phi) \neq 0, \frac{\partial^{2} U}{\partial V^{2}}(\Phi) \neq 0$, and $\frac{\partial^{2} U}{\partial \mathrm{n}^{2}}(\Phi) \neq 0$ for $j=1, \ldots, J$ at any instant $t$, the state of any phase, besides being described by the set of values $\Phi$, can also be described by any of the following sets

$$
\begin{gathered}
\Phi_{\mathrm{v}}(t) \equiv\left\langle\mathrm{S}(t), \frac{\partial U}{\partial \mathrm{V}}(\Phi(t)), \mathrm{n}_{1}(t), \ldots, \mathrm{n}_{J}(t)\right\rangle, \\
\Phi_{\mathrm{s}}(t) \equiv\left\langle\frac{\partial U}{\partial \mathrm{S}}(\Phi(t)), \mathrm{V}(t), \mathrm{n}_{1}(t), \ldots, \mathrm{n}_{J}(t)\right\rangle, \\
\Phi_{\mathrm{nj}}(t) \equiv\left\langle\mathrm{S}(t), \mathrm{V}(t), \mathrm{n}_{1}(t), \ldots, \frac{\partial U}{\partial \mathrm{n}_{\mathrm{j}}}(\Phi(t)), \ldots, \mathrm{n}_{J}(t)\right\rangle, \\
\Phi_{\mathrm{sv}}(t) \equiv\left\langle\frac{\partial U}{\partial S}(\Phi(t)), \frac{\partial U}{\partial \mathrm{V}}(\Phi(t)), \mathrm{n}_{1}(t), \ldots, \mathrm{n}_{J}(t)\right\rangle,
\end{gathered}
$$

among others. Actually, the phase state is described by any one of a family of $2{ }^{+2}$ possible sets of values and, for each set, there is an additive extensive property which is named the thermodynamic potential of the set (Truesdell, 1984). For instance, the thermodynamic potential corresponding to $\Phi_{\mathrm{S}}(t)$ is the Helmholtz energy $\mathrm{A}$ and, from Equation 43 and the definition $\mathrm{A}=U$-TS,

$$
\mathrm{dA}=-\mathrm{SdT}-\mathrm{pdV}+\sum_{j=1}^{J} \mu_{\mathrm{j}} \mathrm{dn}_{\mathrm{j}}
$$

where $\frac{\partial^{2} \mathrm{~A}}{\partial \mathrm{T}^{2}}(\Phi \mathrm{s}) \neq 0, \frac{\partial^{2} \mathrm{~A}}{\partial \mathrm{V}^{2}}(\Phi \mathrm{s}) \neq 0$, and $\frac{\partial^{2} \mathrm{~A}}{\partial \mathrm{n}_{\mathrm{j}}{ }^{2}}(\Phi \mathrm{s}) \neq 0$ for $j=1, \ldots, J$ at any instant $t$.

Analogously, the thermodynamic potential corresponding to $\Phi_{\mathrm{V}}(t)$ is the enthalpy $\mathrm{H}=U+\mathrm{pV}$,

$$
\mathrm{dH}=\mathrm{TdS}+\mathrm{Vdp}+\sum_{j=1}^{J} \mu_{\mathrm{j}} \mathrm{dn}_{\mathrm{j}}
$$


and $\frac{\partial^{2} \mathrm{H}}{\partial \mathrm{S}^{2}}\left(\Phi_{\mathrm{v}}\right) \neq 0, \frac{\partial^{2} \mathrm{H}}{\partial \mathrm{p}^{2}}\left(\Phi_{\mathrm{v}}\right) \neq 0$, and $\frac{\partial^{2} \mathrm{H}}{\partial \mathrm{n}_{\mathrm{j}}{ }^{2}}\left(\Phi_{\mathrm{v}}\right) \neq 0$ for $j=1, \ldots, J$ at any instant $t$. The thermodynamic potential referring to the set $\Phi_{\mathrm{nj}}(t)$ is $Y_{\mathrm{j}}=U-\mu_{\mathrm{j}} \mathrm{n}_{\mathrm{j}}$. By substituting Equation 43 in the expression for $\mathrm{dY}_{\mathrm{j}}$ it follows that

$$
\mathrm{dY} \mathrm{Y}_{\mathrm{j}}=\mathrm{TdS}-\mathrm{pdV}+\mu_{1} \mathrm{dn} \mathrm{n}_{1}+\ldots-\mathrm{n}_{\mathrm{j}} \mathrm{d} \mu_{\mathrm{j}}+\ldots+\mu_{\mathrm{j}} \mathrm{dn} \mathrm{J}_{\mathrm{J}},
$$

and $\frac{\partial^{2} Y_{j}}{\partial S^{2}}\left(\Phi_{n j}\right) \neq 0, \frac{\partial^{2} Y_{j}}{\partial V^{2}}\left(\Phi_{n j}\right) \neq 0, \frac{\partial^{2} Y_{j}}{\partial n^{2}}\left(\Phi_{n j}\right) \neq 0$ for $i=1, \ldots, J$ but $i \neq j$, and $\frac{\partial^{2} Y_{j}}{\partial \mu_{j}{ }^{2}}\left(\Phi_{n j}\right) \neq 0$ at any instant $t$. Finally, the thermodynamic potential corresponding to $\Phi_{\mathrm{SV}}(t)$ is the Gibbs energy $\mathrm{G}=U-\mathrm{TS}+\mathrm{pV}$,

$$
\mathrm{dG}=-\mathrm{SdT}+\mathrm{Vdp}+\sum_{j=1}^{J} \mu_{\mathrm{j}} \mathrm{dn} \mathrm{n}_{\mathrm{j}},
$$

and $\frac{\partial^{2} \mathrm{G}}{\partial \mathrm{T}^{2}}(\Phi \mathrm{sv}) \neq 0, \frac{\partial^{2} \mathrm{G}}{\partial \mathrm{p}^{2}}(\Phi \mathrm{sv}) \neq 0$, and $\frac{\partial^{2} \mathrm{G}}{\partial \mathrm{n}_{\mathrm{j}}^{2}}(\Phi \mathrm{sv}) \neq 0$ for $j=1, \ldots, J$ at any instant $t$. Note that $U$ is the thermodynamic potential corresponding to $\Phi=\left\langle\mathrm{S}, \mathrm{V}, \mathrm{n}_{1}, \ldots, \mathrm{n}_{\rho}\right\rangle$, but $\mathrm{S}$ is not a thermodynamic potential for the set $\left\langle U, V, \mathrm{n}_{1}, \ldots, \mathrm{n}_{J}\right\rangle$, since it is not possible to ensure that the derivative $\frac{\partial^{2} \mathrm{~S}}{\partial \mathrm{V}^{2}}\left(U, \mathrm{~V}, \mathrm{n}_{1}, \ldots, \mathrm{n}_{J}\right)$ is not zero. Thus, the maximization of $\mathrm{S}$ for the stable states of isolated systems does not guarantee that $\mathrm{S}$ is a thermodynamic potential.

\subsection{Temperature}

When the volume and the amount of all substances in the phase do not vary, $U$ is a monotonically increasing function of $S$, and then the partial derivative $\frac{\partial U}{\partial S}(\Phi)$ is a positive quantity. Thus, because this partial derivative is the definition of temperature,

$$
\mathrm{T}=\frac{\partial U}{\partial \mathrm{S}}(\Phi)>0
$$

Because the internal energy is the thermodynamic potential corresponding to the set of values $\Phi, \frac{\partial^{2} U}{\partial S^{2}} \neq 0$ and, to complete the temperature definition, the sign of this second derivative must be stated. In fact, $\frac{\partial^{2} U}{\partial S^{2}}(\Phi)=\frac{\partial T}{\partial S}(\Phi)>0$. Thus, temperature is a concept closely related to the second law of thermodynamics but the first scale of temperature proposed by Kelvin in 1848 emerged as a logical consequence of Carnot's work, without even mentioning the concepts of internal energy and entropy.

Kelvin's first scale includes the entire real axis of dimensionless real numbers and is independent of the choice of the body employed as a thermometer (Truesdell \& Baratha, 1988). The corresponding dimensional scales of temperature are called empirical. In 1854, Kelvin proposed a dimensionless scale including only the positive semi- 
axis of the real numbers. For the corresponding absolute scale (section 2.3), the dimensionless 1 may stand for a phase at $\frac{1}{273.15}$ of the temperature value of water at its triple point. The second scale proposed by Kelvin is completely consistent with the gas thermometer experimental results known in 1854. Moreover, it is consistent with the heat theorem proposed by Nernst in 1905, half a century later.

Because, according to the expression $\frac{\partial \mathrm{T}}{\partial \mathrm{S}}(\Phi)>0$, the variations of temperature and entropy have the same sign, when temperature tends to its maximum lower bound, the same must occur for entropy. But, if the maximum lower bound of entropy is zero as proposed by Planck in 1910, when this value is reached a full knowledge about a state of an isolated homogeneous system should be obtained. Then, because the null absolute temperature is not attainable, another statement could have been made by Planck on Nernst's heat theorem:

"It is impossible to obtain full knowledge about an isolated homogeneous system."

\subsection{Pressure}

In analogy to temperature, pressure is defined by a partial derivative of $U=U\left(\mathrm{~S}, \mathrm{~V}, \mathrm{n}_{1}, \ldots \mathrm{n}_{J}\right)$,

$$
\mathrm{p}=-\frac{\partial U}{\partial \mathrm{V}}(\Phi)
$$

or, alternatively, by

$$
\mathrm{p}=-\frac{\partial \mathrm{A}}{\partial \mathrm{V}}\left(\Phi_{\mathrm{S}}\right)
$$

But, for completing the pressure definition, the signs of the second derivatives of $U$ and $\mathrm{A}$ must be established. Actually, it is easily proved that these second derivatives must have the same sign, so that it is sufficient to state that $\frac{\partial p}{\partial V}(\Phi s)<0$, in agreement with the mechanical concept of pressure. Equation 55 demonstrates that, when $p>0, U$ increases owing to the contraction of phase volume. Hence, according to the principle of conservation of energy, for a closed phase with constant composition and entropy, $p>0$ indicates that the absorption of energy from the outside is followed by volumetric contraction, while $p<0$ implies that absorption of energy from outside is accompanied by volumetric expansion. The former corresponds to an expansive phase tendency, while the latter corresponds to a contractive phase tendency. Evidently, when $\mathrm{p}=0$ no energy exchange between the system and the outside follows volumetric changes. So, the latter corresponds to a non-expansive and noncontractive tendency.

It is clear that $\mathrm{p}$ can assume any value, in contrast to temperature. Hence, the scale for pressure is analogous to Kelvin's first scale, that is, $\mathrm{p}$ can take any real number. For gases, $\mathrm{p}$ is always positive, but for liquids and solids $\mathrm{p}$ can be positive or negative. A stable state of a solid at negative pressure is a solid under tension, but a liquid at negative pressure is in a meta-stable state (Debenedetti, 1996). Thermodynamics imposes no unexpected restriction 
on the value of $\frac{\partial p}{\partial T}\left(\Phi_{s}\right)$ but, because in most cases this derivative is positive, several textbooks consider any stable state presenting a negative value for this derivative as being anomalous. The most well known "anomaly" is related to water, even though there are many others.

\subsection{Chemical potential}

In analogy to pressure, the chemical potential is defined by a partial derivative of $U=U(\mathrm{~S}, \mathrm{~V}$, $\left.\mathrm{n}_{1}, \ldots, \mathrm{n}_{\mathrm{J}}\right)$,

$$
\mu_{\mathrm{j}}=\frac{\partial U}{\partial \mathrm{n}_{\mathrm{j}}}(\Phi)
$$

or, alternatively, by

$$
\mu_{\mathrm{j}}=\frac{\partial \mathrm{G}}{\partial \mathrm{n}_{\mathrm{j}}}(\Phi \mathrm{sv})
$$

Moreover, to complete the chemical potential definition the signs of the second derivatives of $U$ and $G$ must be established. Because these derivatives must have the same sign, it is enough to state that $\frac{\partial \mu_{\mathrm{j}}}{\partial \mathrm{n}_{\mathrm{j}}}\left(\Phi_{\mathrm{sv}}\right)>0$, which illustrates that both $\mu_{\mathrm{j}}$ and $\mathrm{n}_{\mathrm{j}}$ must have variations with the same sign when temperature, pressure and all the other $J-1$ amounts of substance remain unchanged. Remembering that, for the $j \underline{\text { th }}$ chemical species the partial molar value $z_{j}$ of an additive extensive property $z$ is, by definition,

$$
z_{\mathrm{j}}=\frac{\partial z}{\partial \mathrm{n}_{\mathrm{j}}}(\Phi \mathrm{sv}),
$$

Equation 58 shows that $\mu_{\mathrm{j}}=\mathrm{G}_{\mathrm{j}}$, that is, the chemical potential of the $j \underline{t h}$ chemical species is its partial molar Gibbs energy in the phase.

Although $\mu_{\mathrm{j}}$ is called a chemical potential, in fact $\mu_{\mathrm{j}}$ is not a thermodynamic potential like $U, \mathrm{H}, \mathrm{A}, \mathrm{Y}_{\mathrm{j}}$ and $\mathrm{G}$. This denomination is derived from an analogy with physical potentials that control the movement of charges or masses. In this case, the chemical potential controls the diffusive flux of a certain chemical substance, that is, $\mu_{j}$ controls the movement of the particles of a certain chemical substance when their displacement is only due to random motion. In order to demonstrate this physical interpretation, let two distinct but otherwise closed phases with the same homogeneous temperature and pressure be in contact by means of a wall that is only permeable to the $j \underline{t h}$ species. Considering that both phases can only perform volumetric work and are maintained at fixed temperature and pressure, according to Equations 35 and 53

$$
\mathrm{dG}=\mu_{\mathrm{j} 1 \mathrm{dn}} \mathrm{n}_{\mathrm{j} 1}+\mu_{\mathrm{j} 2} \mathrm{dn}_{\mathrm{j} 2} \leq 0,
$$

where the subscripts " 1 " and " 2 " describe the phases in contact. But, because $\mathrm{dn}_{\mathrm{j} 2}=-\mathrm{dn}_{\mathrm{j} 1}$, it follows that 


$$
\left(\mu_{\mathrm{j} 1}-\mu_{\mathrm{j} 2}\right) \mathrm{dn}_{\mathrm{j} 1} \leq 0 .
$$

Thus, $\mathrm{dn}_{\mathrm{j} 1}>0$ implies $\mu_{\mathrm{j} 1}-\mu_{\mathrm{j} 2} \leq 0$, that is, the substance $j$ flows from the phase in which it has a larger potential to the phase in which its chemical potential is smaller.

\section{Conclusion}

By using elementary notions of differential and integral calculus, the fundamental concepts of thermodynamics were re-discussed according to the thermodynamics of homogeneous processes, which may be considered an introductory theory to the mechanics of continuum media. For the first law, the importance of knowing the defining equations of the differentials $\mathrm{dq}, \mathrm{dw}$ and $\mathrm{d} U$ was stressed. Moreover, the physical meaning of $\mathrm{q}, \mathrm{w}$ and $U$ was emphasized and the fundamental equation for homogeneous processes was clearly separated from the first law expression.

In addition, for the second law, a thermally homogeneous closed system was used. This approach was employed to derive the significance of Helmholtz and Gibbs energies. Further, entropy was defined by using generic concepts such as the correspondence between states and microstates and the missing structural information. Thus, it was shown that the concept of entropy, which had been defined only for systems in equilibrium, can be extended to other systems much more complex than the thermal machines. The purpose of this chapter was to expand the understanding and the applicability of thermodynamics.

\section{Acknowledgement}

The authors would like to acknowledge Professor Roy Bruns for an English revision of this manuscript and CNPQ.

\section{References}

Agarwal, R.P. \& O'Regan, D. (2008). Ordinary and Partial Differential Equations: With Special Functions, Fourier Series, and Boundary Value Problems, Springer-Verlag, 978-0-387-79145-6, New York

Apostol, T.M. (1967). Calculus. One-Variable Calculus, with an Introduction to Linear Algebra, John-Wiley \& Sons, 0-471-00005-1, New York

Bassi, A.B.M.S. (2005, a). Quantidade de substância. Chemkeys, (September, 2005) pp. 1-3

Bassi, A.B.M.S. (2005, b). Matemática e termodinâmica. Chemkeys, (September, 2005) pp. 1-9

Bassi, A.B.M.S. (2005, c). Entropia e energias de Helmholtz e de Gibbs. Chemkeys, (November, 2005) pp.1-14

Bassi, A.B.M.S. (2006, a). O conceito de propriedade termodinâmica. Chemkeys, (May, 2006) pp.1-10

Bassi, A.B.M.S. (2006, b). As duas expressões fundamentais da termodinâmica. Chemkeys, (April, 2006) pp.1-8

Boltzmann, L.E. (1964). Lectures on Gas Theory, University of California Press, 0-486-68455-5, Ontario

Brillouin, L. (1962). Science and Information Theory, Academic Press, 0121349500, New York

Casimir, H.B.G. (1945). On Onsager's principle of microscopic reversibility. Review of Modern Physics, Vol. 12, No. 2-3 (April-June, 1945) pp. 343-350, 0034-6861 
Day, W.A. (1987). A comment on a formulation of the second law of thermodynamics. Archive of Rational Mechanics and Analysis, Vol. 98, No. 3, (September, 1987) pp. 211-227, 0003-9527

De Groot, S.R. \& Mazur, P. (1984). Non-equilibrium Thermodynamics, Dover Publications, 0-486-64741-2, Toronto

Debenedetti, P.G. (1996). Metastable liquids: Concepts and Principles, Princeton University Press, 0691085951, Princeton

Eckart, C. (1940). The thermodynamics of irreversible processes I. The simple fluid. Physical Review, Vol. 58, No. 3, (August, 1940) pp. 267-269, 1050-2947

Fermi, E. (1956). Thermodynamics, Dover Publications, 486-60361-X, Mineola

Gray, R.M. (1990). Entropy and Theory Information, Springer-Verlag, 0387973710, New York

Gurtin, M.E. (1971). On the first law of thermodynamics. Archive of Rational Mechanics and Analysis, Vol. 42, No. 2, (January, 1971) pp.77-92, 0003-9527

Hutter, K. (1977). The foundations of thermodynamics, its basic postulates and implications. A review of modern thermodynamics. Acta Mechanica, Vol. 27, No.1-4, (March, 1977) pp. 1-54, 0001-5970

Kestin, J. (1976). The Second Law of Thermodynamics, Halsted Press, 0879332425, New York

Liu, I-Shih. (2002). Continuum Mechanics, Springer-Verlag, 3-540-43019-9, Berlin

Mase, G.T. \& Mase, G.E. (1999). Continuum Mechanics for Engineers, CRC Press, 0-8493-18856, Boca Raton

Mcquarrie, D.A. (2000). Statistical Mechanics, University Science Books, 1891389157, New York.

Michaelides, E.E. (2008). Entropy, order and disorder. The Open Thermodynamics Journal, Vol. 2, No. 2, (March, 2008) pp. 7-11, 1874-396X

Moreira, N.H. \& Bassi, A.B.M.S. (2001). Sobre a primeira lei da termodinâmica. Química Nova, Vol. 24, No. 4, (July-August, 2001) pp. 563-567, 3-540-43019-9

Nery, A.R.L. \& Bassi, A.B.M.S. (2009, a). A quantidade de matéria nas ciências clássicas. Química Nova, Vol. 32, No. 7, (August, 2009) pp. 1961-1964, 1678-7064

Nery, A.R.L. \& Bassi, A.B.M.S. (2009, b). A primeira lei da termodinâmica dos processos homogêneos. Química Nova, Vol. 32, No. 2, (February, 2009) pp. 522-529, 1678-7064

Onsager, L. (1931, a). Reciprocal relations in irreversible processes I. Physical Review, Vol.37, No.4, (February, 1931) pp. 405-426, 1050-2947

Onsager, L. (1931, b). Reciprocal relations in irreversible processes II. Physical Review, Vol. 38, No. 12, (December, 1931) pp. 2265-2279, 1050-2947

Planck, M. (1945). Treatise on Thermodynamics, Dover Publications, 048666371X, New York

Serrin, J. (1979). Conceptual analysis of the classical second law of thermodynamics. Archive of Rational Mechanics and Analysis, Vol. 70, No. 4, (December, 1979) pp. 355-371, 0003-9527

Šilhavý, M. (1983). On the Clausius inequality. Archive of Rational Mechanics and Analysis, Vol. 81, No. 3, (September, 1983) pp. 221-243, 0003-9527

Šilhavý, M. (1989). Mass, internal energy and Cauchy's equations of motions in frame-indifferent thermodynamics. Archive of Rational Mechanics and Analysis, Vol. 107, No. 1, (March, 1989) pp. 1-22, 0003-9527

Šilhavý, M. (1997). The Mechanics and Thermodynamics of Continuous Media, Springer-Verlag, 3-540-58378-5, Berlin 
Tolman, R.C. (1938). The Principles of Statistical Mechanics, Oxford Press, 0-486-63896-0, New York

Toupin, R. \& Truesdell, C.A. (1960). The classical field of theories, In: Handbuch der Physik, S. Flügge (Ed.), pp. 226-858, Springer-Verlag, 0085-140X, Berlin

Truesdell, C.A. \& Baratha, S. (1988). The Concepts and Logic of Classical Thermodynamics as a Theory of Heat Engines: Rigorously Constructed upon the Foundation Laid by S. Carnot and F. Reech, Springer-Verlag, 3540079718, New York

Truesdell, C.A. (1980). The Tragicomical History of Thermodynamics, 1822-1854, Springer-Verlag, 0-387904034, New York

Truesdell, C.A. (1984). Rational Thermodynamics, Springer-Verlag, 0-387-90874-9, New York

Truesdell, C.A. (1991). A First Course in Rational Continuum Mechanics, Academic Press, 0127013008, Boston

Williams, W.O. (1971). Axioms for work and energy in general continua. Archive of Rational Mechanics and Analysis, Vol. 42, No. 2, (January, 1972) pp. 93-114, 0003-9527 


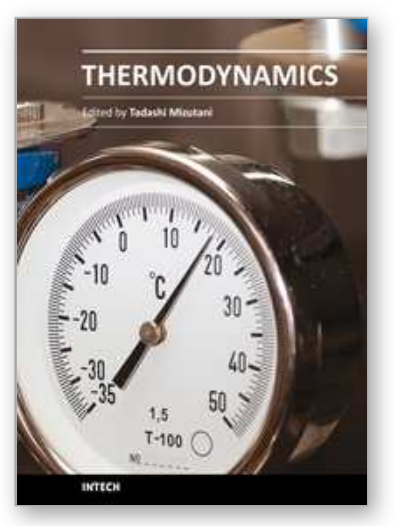

\author{
Thermodynamics \\ Edited by Prof. Mizutani Tadashi
}

ISBN 978-953-307-544-0

Hard cover, 440 pages

Publisher InTech

Published online 14, January, 2011

Published in print edition January, 2011

Progress of thermodynamics has been stimulated by the findings of a variety of fields of science and technology. The principles of thermodynamics are so general that the application is widespread to such fields as solid state physics, chemistry, biology, astronomical science, materials science, and chemical engineering. The contents of this book should be of help to many scientists and engineers.

\title{
How to reference
}

In order to correctly reference this scholarly work, feel free to copy and paste the following:

Martina Costa Reis and Adalberto Bono Maurizio Sacchi Bassi (2011). On the Two Main Laws of Thermodynamics, Thermodynamics, Prof. Mizutani Tadashi (Ed.), ISBN: 978-953-307-544-0, InTech, Available from: http://www.intechopen.com/books/thermodynamics/on-the-two-main-laws-of-thermodynamics

\section{INTECH}

open science | open minds

\section{InTech Europe}

University Campus STeP Ri

Slavka Krautzeka 83/A

51000 Rijeka, Croatia

Phone: +385 (51) 770447

Fax: +385 (51) 686166

www.intechopen.com

\section{InTech China}

Unit 405, Office Block, Hotel Equatorial Shanghai

No.65, Yan An Road (West), Shanghai, 200040, China

中国上海市延安西路65号上海国际贵都大饭店办公楼405单元

Phone: +86-21-62489820

Fax: $+86-21-62489821$ 
(C) 2011 The Author(s). Licensee IntechOpen. This chapter is distributed under the terms of the Creative Commons Attribution-NonCommercialShareAlike-3.0 License, which permits use, distribution and reproduction for non-commercial purposes, provided the original is properly cited and derivative works building on this content are distributed under the same license. 